\title{
Zero-Point Energy and the Emergence of Gravity: Two Hypotheses
}

\author{
Philip J. Tattersall ${ }^{1}$ \\ ${ }^{1}$ Private researcher, 8 Lenborough St, Beauty Point, Tasmania, Australia \\ Correspondence: Philip J. Tattersall, 8 Lenborough St, Beauty Point, Tasmania, Australia. Email: \\ soiltechresearch@bigpond.com
}

Received: February 16, 2017

Accepted: March 10, $2017 \quad$ Online Published: March 20, 2017

doi:10.5539/apr.v9n2p72

URL: https://doi.org/10.5539/apr.v9n2p72

\begin{abstract}
Building on the results of experiments and speculations arising from his earlier collaborations as well as proposals from the theoretical work of others, the author suggests that gravity emerges as a result of the interaction of zero-point energy with matter. The results of experiments suggest that light, through its interaction with zero-point energy causes changes in the weight of test masses. These experimental results are interpreted as being due to changes in the spectral [Note 1] content surrounding massive bodies. Two hypotheses therefore emerge. Further experiments are planned to test the findings from earlier experiments.
\end{abstract}

Keywords space, falling frames, zero-point, ZPF, ZPE, gravity, vacuum, electromagnetic quantum vacuum, general relativity, Stochastic Electrodynamics (with spin), SED, SEDS pressure, relativity, GR

\section{Introduction}

Over many years the author has reflected on the nature of gravity, inertia and mass. A further impetus for work on the part of the author arose from the thought provoking paper by Sir Michael Atiyah presented at the 2005 Einstein lecture on the nature of space (Johnson \& Walker, 2005). Sir Michael speculated that understanding space would be very much central in efforts to unravel many of the outstanding questions within physics. Johnson and Walker (2005) reported that Sir Michael asserted that “...understanding space is the fundamental problem of physics, and his talk focused extensively on the relationship between mathematics and physics, particularly with regard to the nature of space.". Over the years many researchers have been preoccupied with the true nature of the emptiness that is space and its connection to the problem of gravity, among them Sakharov (1967), Zel'dovich (1967), Misner, Thorn and Wheeler (1971), Puthoff (2001), and Wüthrich (2005) to name a few.

As discussed in an earlier paper (Tattersall, 2016) space is considered to be full of a special form of energy known as zero-point energy or vacuum quantum fluctuations. Tattersall (2016) states, “...These speculations (along with the theoretical underpinnings of SED) would lead one to suggest that space is not full of ZPF, but rather that space is in fact ZPF. This is not a return to the aether theory of old, but is an acknowledgement of a mounting body of theoretical argument and experimental work suggesting that 'space' is in fact an active, all pervasive medium." This line of thinking emerged from reflections on the work of Santos (2012), in which he discussed the significance of zero-point energy fluctuations in correlation of quantum phenomena at different spatial points.

A suggestion, put forward by Santos (2012) in which he alluded to the possibility that zero-point energy may be responsible for maintaining the electron stability [Note 2] within atoms via a resonance-type interactions led the author to reexamine the theoretical work of Haisch, Rueda, and Puthoff (1997), Puthoff (2001), Rueda and Haisch (2005). This along with the findings of Rancourt (2011) and Rancourt and Tattersall (2015) led to an interesting conclusion, particularly when entertaining new ideas as to the possible nature of gravity as a pushing force or pressure.

In recent years collaboration between Rancourt and Tattersall (2015) produced interesting and puzzling results which raised some interesting questions and speculations. One speculative conclusion arising from that work suggested that gravity is the result of a 'pushing force' or pressure resulting from some kind of energetic influence originating in the surrounding space. The idea of gravity as a 'pressure force' is not new (Arminjon, 1996). A concept of 'ether' has been proposed, with properties consistent with certain interpretations of special relativity (the Lorentz-Poincaré interpretation) (Arminjon, 1996). This line of thinking is consistent with the emerging ideas on the part of the author as outlined above, namely that space is not just full of ZPE activity, but that space is ZPE activity. 
This paper, in similar style to a previous paper (Tattersall, 2016), takes a phenomenological road and in doing so echoes many of the sentiments expressed in Serge's (2016) paper. He states, “...it becomes clear that the understanding of the whole complex of the indicated problems [in physics], as well as the nature of gravity, should be searched for not on the road of creation of new theories, but on the level of phenomenology. Indeed, phenomenology (phenomenon - the manifesting: logos - the concept doctrine) can become a starting point for (He quotes Heidegger); 'the elucidation of the reality exactly as it shows itself before the science turns to it with its questions"'. Serge (2016) then continues, "Phenomenology can be regarded as a general realistic methodology of cognizing the essence of this or that phenomenon...".

This paper takes the view that efforts to elucidate of the essence of gravity should take advantage of aspects of the newer approaches to scientific inquiry foreshadowed by David Bohm (Tattersall \& Sidebottom, 2014). Well thought through speculations essentially take the form of what could be regarded as pre-hypotheses. The emergence of such special speculations may well take many forms as part of creative-imaginative processes that sit at the very heart of the scientific project. Science, like art generally, bears the indelible signature of our humanness (Tattersall, 2015).

\section{Gravity and Quantum Vacuum (Zero Point Energy)}

Rueda and Haisch (2005), during a discussion on the physical basis of the principle of equivalence, successfully make use of Einstein's falling frames concept in analyzing the physical basis of the principle of equivalence (the equivalence between inertial and gravitational mass). They state, "...The interaction between the electromagnetic quantum vacuum and the electromagnetically-interacting particles constituting any physical object (quarks and electrons) is identical for the two situations of (a) accelerating with respect to constant velocity inertial frames or (b) remaining fixed above some gravitating body with respect to freely falling inertial frames." Holding a body stationary with respect to freely falling frames leads to the force we term weight. Otherwise freely falling bodies experience weightlessness as they undergo acceleration due to the 'pull' of gravity (Newton) or the curvature of space-time (Einstein). Having clearly shown the consistency of their quantum vacuum inertia hypothesis with the primary tenets of general relativity Rueda and Haisch (2005) acknowledge that the origin of active gravitation mass is yet to be explicated in terms of their quantum vacuum inertia hypothesis.

In introducing the freely falling frame concept in this way Rueda \& Haisch have, in the author's view made a highly significant and important contribution to a solution to the gravity 'problem'. The present paper aims to link selected ideas of Santos (2012) with those of Rueda and Haisch and in so doing seeks to point to an apparently simple solution. The theoretical review papers of Margan (nd (a) \& nd (b)) are also acknowledge as playing a significant and influential role in the clarification of the thinking on the part of the author.

\section{Zero-Point Energy (Quantum Vacuum Energy) as a Freely Falling Frame}

Rueda and Haisch (2005) had considered the idea of quantum vacuum energy continually falling into a massive object (say a planet). They posited that a first intuition might suggest that a continual influx of vacuum energy into a massive body would lead to a steady increase of internal energy with time. Their discussion correctly concluded that there is no real change in energy content of the massive body as a result of the entry of an energy flux within falling frames. Of course based purely on the kinematical considerations a stationary observer will reach a different conclusion to that of the freely falling observer. Rueda and Haisch (2005) state, "...for an observer on the surface of the Earth there is no real transfer of energy from the vacuum to objects on the surface of the Earth. In this respect the energy growth...that a freely falling observer sees as time progresses, is purely a kinematical effect that comes from the fact that in the freely-falling frame the velocity of the object is growing with time and does not vanish except at the instant of coincidence..."

\subsection{Experimental Results Suggest the Reality of 'Falling Frames'}

The experiments of Rancourt (2011), Rancourt and Tattersall (2015) found that depending on the position of selected light sources, test masses would register differing weights under carefully controlled experimental conditions. These results pointed to the influx of some kind of energetic influence into matter. It appeared that somehow light was interacting with gravitation 'pressure'. These findings led the author to reconsider falling frames as perhaps real and that gravity may in fact emerge from the pressure created by the influx of zero-point energy into matter. As discussed in a previous paper (Tattersall, 2016), zero-point energy in the form of a field is hypothesized to be moving into matter. Specifically it was stated that, "Let us speculate that the ZPF is continually moving into matter in order to provide an energy source for electron activity and possibly other activity within atoms. It would follow that the intensity of movement of the ZPF would be proportional to both mass and distance. The net result would be a distortion [or perhaps density and pressure] of space in the vicinity of massive bodies. On the face of it the mathematical approach used in GR would still apply." (bolded text added emphasis by author). These considerations led in turn to further reflections on the nature of gravity in terms Stochastic Electrodynamics (SED) and its next generation Stochastic Electrodynamics with Spin (SEDS) [Note 3]. However it later became 
clear that the idea of 'falling frames' of ZPE as a cause of gravity was a surrogate for a deeper and more profound explanation.

\section{The Nature of Gravity - Pointers to Causation}

The cogent arguments offered by Rueda and Haisch regarding freely falling frames confirm the original reasoning employed by Einstein as a way of generalizing the key tenants of special relativity in terms of accelerating frames of reference. It turns out that their use in explicating the cause of gravitation is of limited value as the reality of the distortion of space-time in the vicinity of matter still warrants explanation. As discussed earlier, much work is underway by the physics community on that project. For instance work continues in the areas of general relativity (GR) and quantum theory as researchers work towards unification of the two theoretical frameworks. Other researchers such as Verlinde (2010) are developing explanations along the lines of information theory enshrined in the 'holographic principle'. We take the view that while such theoretical frameworks can provide very accurate predications (e.g. GR) and convincing explanations they are largely silent regarding fundamental causes.

In terms of the present paper we are confronted by a dilemma: An elegant theoretical explanation of the effects of gravity, including a powerful ability to predict on the one hand, while on the other we have before us puzzling experimental findings that appear to point toward some kind of interaction of light with gravity, or perhaps with those energies that are the cause of gravity. More than ever there is justification to move beyond prediction and observation of effects and to attempt to address causes.

It is plausible that the interaction of energies within the zero-point field in the vicinity of matter cause changes in local energy density and therefore its gradient. It has been proposed by E. Margan [Notes $4 \& 5$ ] that both virtual and real photons interact with the 'real' particles forming a massive body, some being scattered, some absorbed and re-emitted and some reflected. The result in general is an altered spectral [Note 1] content surrounding the body and as a consequence a decreased energy density, the emergent effect being what we term 'gravity'. In a sense then bodies are forced together as a result of an energy pressure differential. This line of reasoning leads to an interesting conclusion in light of recent experiments (Rancourt \& Tattersall, 2015).

\subsection{Interpretations from Experimental Findings}

In the case of the Rancourt and Tattersall (2015) experiments test masses suspended under a light source recorded a loss in weight, whereas those masses suspended above the light source recorded a gain in weight. These results suggest that electromagnetic energy present between a massive body $\mathrm{W}$ and test body $\mathrm{w}(\mathrm{W}>>\mathrm{w})$ increases gravitation 'force', whereas the same light source above a test mass w causes a decreased gravitational force. One interpretation would suggest that depending on its location with respect to the large and small masses the light source is somehow causing changes in ZPF pressure density. It appears as though pressure effects originating in the light source itself are responsible i.e. the light source appears to be 'attracting' w, thus changing its weight. Using E. Margan's speculation, as well as the theoretical reasoning of Arminjon (1996), regarding energy density in the vicinity of mass, it could be that the ZPF energy density present in the light source creates a pressure asymmetry into which the nearby mass $(w)$ is forced. This could explain the positive and negative changes in weight of w. Considerations along similar lines were also discussed by Serge (2016).

It is important to point out that the suggestion presented here that zero-point energy is solely the source of the influence is only provisional as other entities may be responsible either alone or in concert with ZPF energies. In other words the energetic influence may not be exclusively electromagnetic (EM) in origin as other fields may also play a role, either alone or in concert with EM fields.

\section{Further Experiments About to Commence}

Hardware is now under construction in which light will be reflected multiple times in a specially designed light box similar to those reported in an earlier experiment (Rancourt \& Tattersall, 2015). In the new experiments an 80000 lumen laminar beam will be reflected in zig-zag fashion through several mirrored layers. The overall experimental setup will be similar to that employed in the 2015 experiments [Note 6].

\section{Conclusions}

The experiments (Rancourt, 2011; Rancourt \& Tattersall, 2015) and their interpretation point to a proposal put forward by E. Margan regarding a possible mechanism for the emergence of what is termed gravity. Changes in the spectral [Note 1] characteristics in the ZPE field surrounding a massive body are thought to be responsible for the emergence of a pressure effect (gravity). The experimental findings, although tentative, suggest that this is in fact the case.

While these hypotheses require further analysis and perhaps development, subsequent experimental work may contribute to a better understanding of gravity, inertia and mass. Serge (2016) has suggested further experiments 
are needed based on the published experimental results of Rancourt \& Tattersall (2015).To that end Rancourt and Tattersall (2015) will soon undertake further experimental work in a bid to test and verify their earlier findings.

\section{References}

Arminjon, M. (1996). Gravitation as a pressure force: a scalar ether theory. Proc. $5^{\text {th }}$ International Conference, Physical Interpretations of Relativity Theory. British Soc. Philos. Sci./University of Sunderland. Retrieved from https://arxiv.org/ftp/arxiv/papers/1112/1112.1875.pdf

Cavalleri, G., Barbero, F., Bertazzi, G., Cesaroni, E., Tonni, E., Bosi, L., ... Gillies, G. T. (2010). A quantitative assessment of stochastic electrodynamics with spin (SEDS): physical principles and novel applications. Frontiers of Physics in China, 5(1).

Haisch, B., Rueda, A., \& Puthoff, H. E. (1997). Physics of the zero-point field: implications for inertia, gravitation and mass. Speculations in Science and Technology, 20.

Johnson, G. W., \& Walker, M. E. (2005). Sir Michael Atiyah's Einstein lecture: “The Nature of Space". Notices of the AMS, 53(6).

Margan, E. (nd(a)). Estimating the vacuum energy density - an overview of possible scenarios, Experimental Particle Physics Department. Jožef Stefan, Institute, Ljubljana, Slovenia.

Margan, E. (nd(b)). Some intriguing consequences for the quantum vacuum fluctuations in the semi-classical formalism. Experimental Particle Physics Department. Jožef Stefan, Institute, Ljubljana, Slovenia.

Marshall, T. W., \& Santos, E. (1997). The myth of the photon. Retrieved from https://arxiv.org/abs/quant-ph/9711046\#

Misner, C. W., Thorne, K. S., \& Wheeler, J. A. (1971). Gravitation. New York. Freeman.

Puthoff, H. E. (2001). Polarizable-vacuum (PV) representation of General Relativity. Retrieved from http://lanl.arxiv.org/ftp/gr-qc/papers/9909/9909037.pdf

Rancourt, L. (2011). Effect of light on gravitational attraction. Physics Essays, 24(4). Retrieved from https://physicsessays.org/browse-journal-2/product/212-16-pdf-louis-rancourt-effect-of-light-on-gravitationalattraction.html

Rancourt, L., \& Tattersall, P. J. (2015). Further experiments demonstrating the effect of light on gravitation. Applied Physics Research, 7(4), 4-13. http://dx.doi.org/10.5539/apr.v7n4p4

Rueda, A., \& Haisch, B. (2005). Gravity and the quantum vacuum inertia hypothesis. Ann. Phys. (Leipzig), 14(8). Retrieved from http://onlinelibrary.wiley.com/doi/10.1002/andp.200510147/full

Sakharov, A. D. (1967). Vacuum quantum fluctuations in curved space and the theory of gravitation. General Relativity and Gravitation, 32(2), 2000

Serge, T. (2016). On the law of universal gravitation and the quantum essence of gravity: phenomenology. Astronomy and Space Science: Open Access, 1(1). Retrieved from http://crescopublications.org/pdf/ ASSOA/ASSOA-1-003.pdf

Santos, E. (2012). Vacuum fluctuations, the clue for a realistic interpretation of quantum mechanics. Retrieved from https://arxiv.org/pdf/1208.4431.pdf

Tattersall, P. J. (2015). Not So Full of Emptiness... (Personal Reflections and Speculations on the Nature and Significance of Space). Resource Publications. Beauty Point, Tasmania, Australia. Retrieved from www.resource-publications.com.au

Tattersall, P. J. (2016). On the nature of space and the emergence of the new physics: some speculations. Applied Physics Research, 8(6). Retrieved from http://dx.doi.org/10.5539/apr.v8n6p25

Tattersall, P. J., \& Sidebottom, B. P. (2014). Towards A new philosophical foundation for physics - speculations on the nature of space, gravity, inertia and mass. Advances in Systems Science and Applications, 14(1). Retrieved from http://metadesigners.org/

Verlinde, E. P. (2010). Origin of Gravity and the Laws of Newton. Retrieved from https://arxiv.org/pdf/ 1001.0785.pdf

Wüthrich, C. (2005). To quantize or not to quantize: fact and folklore in quantum gravity. Philosophy of Science, $72,777-788$.

Zel'dovich, Y. B. (1967). JETP letters, 6, 316. Soviet Physics Uspekhi, 11(381). 


\section{Notes}

Note 1. The term spectral is used with caution herein as the nature of gravity is as yet unknown. While it would seem that an energy density does exist its actual physical make-up is yet to be characterised. Current physical theory would demand that even if the energy was to be in the form of ZPE it would by necessity have a spectral form.

Note 2. This refers to the electron itself where its internal energy oscillations must be in balance with the external energy density (a 'pressure'). Matter then is a kind of self-resonance of the trapped energy and the external energy. (E. Margan, Personnel communication, February 26, 2017).

Note 3. SEDS is an important development and has been discussed at length by Cavalleri et al (2009). The significance of their review is far reaching. Introducing spin into SED overcomes many of its shortcomings and indeed brings clarity to many of the inexplicable aspects of QED, including the physical origin of ZPF, the neutrino rest mass anomaly and the high energy tail of cosmic rays to name a few. Cavalleri et al (2009) propose experiments they believe are capable of discriminating between QED and SEDS. The paradoxical results of the double slit experiment are discussed and a tentative explanation is offered under SEDS where the ZPF is implicated. There are echoes of the work of T. W. Marshall \& E. Santos (Marshall \& Santos, 1997), in which they show that modes within the ZPF interact with photons.

In terms of the present paper we believe that the theoretical developments coming out of SEDS will assist us in the interpretation of the experimental findings involving the effect of light on the spectral content surrounding mass, even though the light itself does not appear to physically contact test masses.

Note 4. Personal communication from Erik Margan, Experimental Physics Department, Jožef Stefan, Institute, Ljubljana, Slovenia.

Note 5. An acknowledgement of the collegial encouragement of Erik Margan for his support in reviewing drafts of this paper and for his supportive suggestions and comments over several weeks during which we corresponded on many occasions.

Note 6. The experimental development for the new setup is discussed at https://www.researchgate.net/project/ Levitation-of-a-100-g-mass-using-blue-laser-light

\section{Copyrights}

Copyright for this article is retained by the author(s), with first publication rights granted to the journal.

This is an open-access article distributed under the terms and conditions of the Creative Commons Attribution license (http://creativecommons.org/licenses/by/4.0/). 